\title{
Neuartiger thermischer Wirbelsensor zur Bestimmung von binären Mischungsverhältnissen in Fluiden
}

\author{
B. Schmitt, C. Kiefer, A. Schütze \\ Universität des Saarlandes, Lehrstuhl für Messtechnik, Campus A5 1, Saarbrücken, Deutschland \\ b.schmitt@LMT.uni-saarland.de
}

\begin{abstract}
Zusammenfassung:
Ein neuartiger thermischer Sensor zur Bestimmung von Mischungsverhältnissen wird vorgestellt. Das Messprinzip beruht auf der Nutzung eines stationären Wirbelpaares, das sich bei geringen Reynoldszahlen hinter einem in einer Strömung befindlichen Störkörper bildet. Die Ausdehnung des Wirbels in Strömungsrichtung ändert sich unter anderem mit der Viskosität des anströmenden Gemisches, wobei durch ihre Bestimmung bzw. Auswertung ihrer Änderung auf das Mischungsverhältnis geschlossen werden kann. Mikrostrukturierte Heizer werden in dem Totgebiet hinter dem Störkörper platziert, um dieses zu erfassen, was eine bessere Auflösung des Mischungsverhältnisses ermöglicht als dies in der freien Strömung möglich wäre. In ersten Messungen von Methanol/Wasser-Gemischen konnten bei einer Flussrate von 0,09 ml/s Auflösungen von 0,5 Vol.-\% erreicht werden. Die einfache und kostengünstige Herstellung der Sensoren und der Messkammer verspricht ein großes Einsatzpotential des Messprinzips, beispielsweise in Direktmethanolbrennstoffzellen zum Betrieb von mobilen Geräten.
\end{abstract}

Key words: Thermischer Wirbelsensor, stationäres Wirbelpaar, Mischungsverhältnis, Direktmethanolbrennstoffzelle, Selective Catalytic Reduction.

\section{Einleitung}

In vielen technischen Systemen ist die Überwachung und Regelung des Mischungsverhältnisses von Flüssigkeiten maßgebend für die ordnungsgemäße Funktion. Der Einsatzbereich reicht dabei von der Messung von Methanol in Wasser für Direktmethanolbrennstoffzellen (engl. Direct Methanol Fuel Cell, DMFC) über Harnstoff in Wasser für die Abgasnachbehandlung von Dieselfahrzeugen mittels selektiver, katalytischer Reduktion (engl. Selective Catalytic Reduction, SCR) bis hin zur Überwachung des Frostschutzmittelanteils in Kühlkreisläufen.

DMFCs sind aufgrund ihrer kleinen Baugröße gerade für den Einsatz in mobilen Geräten wie Laptops prädestiniert, um deren Laufzeit im Vergleich zur Nutzung konventioneller Akkumulatoren zu verlängern [1]. Allerdings muss der Zelle zur Erreichung eines optimalen Wirkungsgrades je nach Typ eine konstante Methanolkonzentration von 1,2 bis 4,1 Vol.- $\%$ zugeführt werden [2,3]. Diese nimmt in der Zelle ab, wobei die optimale Konzentration im Kreislauf durch Beimengen von reinem Methanol wieder hergestellt werden soll, sodass sie der Zelle erneut zugeführt werden kann. Hierbei muss die Methanolkonzentration mittels eines Sensors erfasst und anschließend ihre Verringerung ausgeglichen werden.
Bei der Abgasnachbehandlung von Dieselfahrzeugen mittels SCR wird eine wässrige Lösung aus 32,5 Gew.-\% Harnstoff vor dem DeNOxKatalysator in den Abgasstrang eingespritzt. Dort bildet sich aus dem Harnstoff aufgrund der hohen Temperaturen durch Thermo- und Hydrolyse Ammoniak, das mit den Stickoxiden zu harmlosem Stickstoff und Wasser reagiert. Dies ist die einzige derzeit bekannte Methode zur sicheren Einhaltung der Abgasgrenzwerte von Dieselfahrzeugen bei gleichzeitiger Verbesserung des Leistungsverhaltens des Motors [4]. Zur Vermeidung einer Fehlbetankung des separaten Tanks der Harnstoffmischung mit z.B. Leitungswasser oder Dieselkraftstoff wird hierbei ein Sensor benötigt, der die Qualität der Mischung überwacht.

Diesen exemplarischen Einsatzbereichen ist gemein, dass ein wichtiger Fokus des möglichen Sensorprinzips auf der kostengünstigen Herstellung und Auswertung liegt. Bereits in früheren Arbeiten wurde ein thermisches Sensorprinzip präsentiert, mit dem dies bei ausreichender Genauigkeit der Bestimmung des Mischungsverhältnisses möglich war [5]. Dabei wurden die unterschiedlichen, thermischen Eigenschaften der bekannten Komponenten in einer Mischung genutzt, die den Wärmeabtransport eines eingeprägten Wärmepulses beeinflussen. In der hier dargestellten Arbeit 
wird ein in der Mischung befindlicher Störkörper genutzt, der bei geringen Strömungsgeschwindigkeiten ein stationäres Wirbelpaar erzeugt, wobei die Charakterisierung von dessen Totgebiet eine Verbesserung der Auflösung des Mischungsverhältnisses ermöglicht.

\section{Messprinzip}

Durch Einbringen eines Störkörpers in eine Flüssigkeitsströmung ergeben sich je nach Randbedingung verschiedene Umströmungen [6]. Diese führen bei sehr kleinen Reynoldszahlen $R e$ zunächst zu einer schleichenden Umströmung des Störkörpers. Mit zunehmendem $R e$ bildet sich ein stationäres, symmetrisches Wirbelpaar aus, die ein Totgebiet hinter dem Störkörper erzeugen (siehe Abb. 1). Bei weiter steigenden Reynoldszahlen lösen sich die beiden Wirbel abwechselnd vom Störkörper, was schließlich als laminare oder Kármánsche Wirbelstraße bekannt ist $[6,7]$.

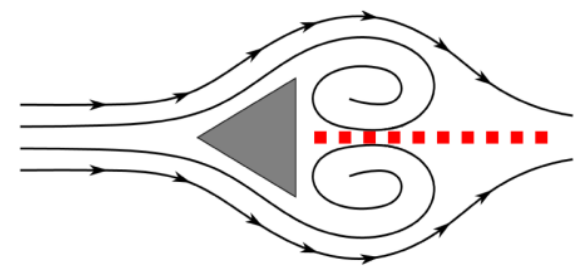

Abb. 1. Schematische Darstellung eines stationären Wirbelpaares hinter einem keilförmigen Störkörper mit in Rot dargestelltem Heizerarray.

Letztere hat sich bereits in der Nutzung in Strömungssensoren (sog. Wirbelzähler bzw. Vortex-Durchflussmesser) etabliert, wobei die Wirbelablösefrequenz $f$ abhängig von der Strouhalzahl St des umströmten Körpers, seiner charakteristischen Länge $d$ und der Anströmgeschwindigkeit $u_{0}$ ist [7]:

$$
f=S t \cdot \frac{u_{0}}{d}
$$

Das neue Messprinzip macht sich die räumliche Ausdehnung des stationären Wirbelpaares in Strömungsrichtung zunutze, das im Falle von zylindrischen Störkörpern bei relativ kleinen Reynoldszahlen $R e$ linear abhängig von diesen ist [6]. Da $R e$ neben der Anströmgeschwindigkeit und der charakteristischen Länge $d$ des Störkörpers die kinematische Viskosität $v(\phi, T)$ des anströmenden Gemischs umfasst (siehe GI. (2) [7]), kann somit bei Kenntnis von Strömungsgeschwindigkeit und Temperatur durch Messung der Ausdehnung des Wirbels auf die Viskosität des Gemischs und dadurch auf die Mischungsanteile $\phi_{i}$ der bekannten Komponenten $i=1,2$ geschlossen werden. Umgekehrt ist es auch möglich, bei bekannter Viskosität die Geschwindigkeit der anströmenden Flüssigkeit zu bestimmen. Abb. 2 zeigt die kinematische
Viskosität für Methanol/Wasser-Gemische in Abhängigkeit der Volumenkonzentration von Methanol bei verschiedenen Temperaturen.

$$
R e=u_{0} \frac{d}{v\left(\Phi_{i}, T\right)}
$$

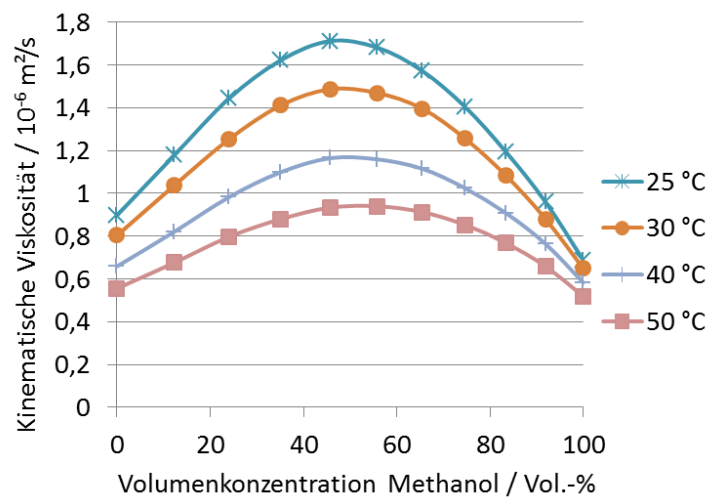

Abb. 2. Kinematische Viskosität von Methanol/ Wasser-Gemischen in Abhängigkeit der Volumenkonzentration von Methanol und der Temperatur (Werte berechnet aus dyn. Viskosität und Dichte [8]).

Die Messung der Wirbelausdehnung erfolgt hierbei mittels eines hinter dem Störkörper befindlichen Heizerarrays, siehe Abb. 1. Werden die Heizer mit einem elektrischen Strom beaufschlagt, so erwärmen sie sich, wobei Wärme zusätzlich zur Wärmeleitung aufgrund der Strömung durch erzwungene Konvektion abgeführt wird. Diese Abkühlung ist umso stärker, je höher die lokale Strömungsgeschwindigkeit [9] ist. Da das Gemisch hinter dem Störkörper in entgegengesetzter Strömungsrichtung fließt, existiert ein Punkt am Ende des Wirbels, in dem die Strömungsrichtung umschlägt und dadurch die Strömungsgeschwindigkeit gerade Null ist. Ein Heizer an dieser Position erfährt somit weniger Abkühlung aufgrund von Konvektion, wodurch er im Verhältnis zu den benachbarten Heizern die höchste Temperatur erreicht. Die Position dieses Heizers gibt somit die Wirbelausdehnung an und lässt den Rückschluss auf die Viskosität und damit auf das Mischungsverhältnis zu.

Das Messprinzip wurde in Finite Elemente Simulationen unter Nutzung vom Comsol Multiphysics ( $\mathrm{Fa}$. Comsol $\mathrm{AB}$ ) simuliert. Abb. 3 zeigt im Falle von Wasser die mittig hinter dem keilförmigen Störkörper in Strömungsrichtung auftretenden Geschwindigkeiten bei verschiedenen Flussraten in Abhängigkeit vom Abstand zum Störkörper. Der Querschnitt der Zuleitung beträgt $1 \times 1 \mathrm{~mm}^{2}$ (vgl. Abb. 5). So ist bei geringen Flussraten zu erkennen, dass bei einem Abstand zum Keil von unter ca. $2 \mathrm{~mm}$ die Fließgeschwindigkeit verringert ist und sich bei größeren Abständen stabilisiert. Bei Flussraten 
über $0,03 \mathrm{ml} / \mathrm{s}$ hingegen ergibt sich bedingt durch das stationäre Wirbelpaar eine Strömung in entgegengesetzter Richtung. Die Wirbelausdehnung wächst mit der Geschwindigkeit und ist am Nulldurchgang des Verlaufs ablesbar.

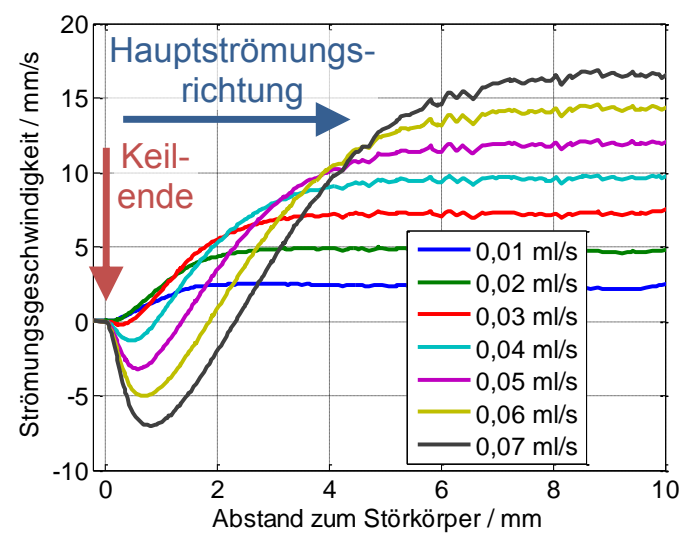

Abb. 3. Simulation der hinter einem Störkörper (vgl. Abb. 5) vorliegenden Geschwindigkeiten für verschiedene Flussraten von Wasser.

\section{Messaufbau}

Die zur Verifizierung des Messprinzips entworfene Messkammer ist in Abb. 4 dargestellt. Zum einfachen Testen verschiedener Geometrien der Störkörper wurden die Einsätze mittels 3D-Druck hergestellt (Connex 500, Fa. Objet), die in die Messkammer eingebaut und leicht ausgetauscht werden können (siehe Abb. 5).

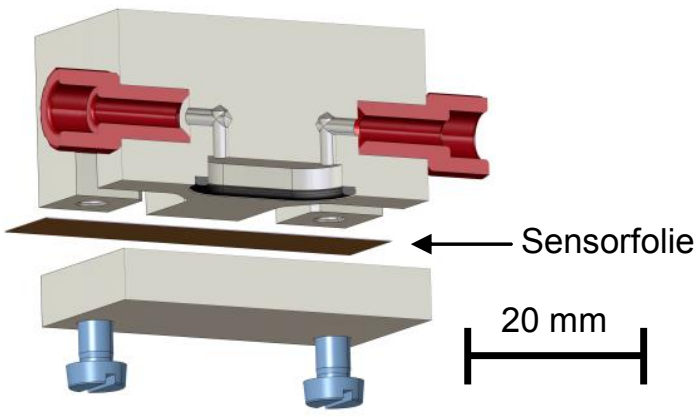

Abb. 4. 3D-Schnittdarstellung der genutzten Messkammer. In den Hohlraum wird ein Einsatz eingesetzt, der dann von der Mischung durchströmt wird.

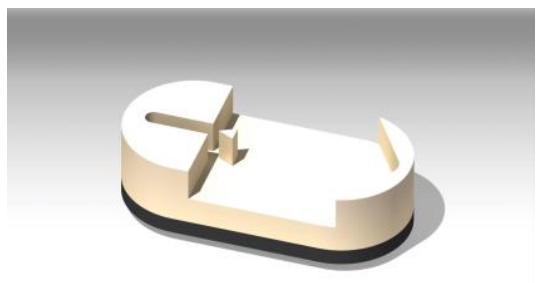

Abb. 5. Mittels 3D-Druck erzeugter Einsatz mit keilförmigem Störkörper (Grundfläche: 1x1 mm²) zum Einbau in die Messkammer. Rückseitig ist eine fest verbundene Dichtung aufgedruckt. Am Anfang des Einströmkanals und am Ende des Einsatzes sind jeweils Öffnungen für den $\mathrm{Zu}$ - und Abfluss.
Der genutzte Drucker bietet zudem die Möglichkeit, verschiedene, unterschiedlich elastische Verbundmaterialien zu drucken, wodurch die Einsätze mit einer fest verbundenen Dichtung an der Rückseite hergestellt werden konnten.

Das Heizerarray wurde auf einer Kapton-Folie mit mikrostrukturierten Heizern aus Aluminium realisiert. Die Folie mit den darauf befindlichen Heizern und zusätzlichen Temperatursensoren wird durch die Bodenplatte der Messkammer auf den Einsatz gepresst und vom zu vermessenden Flüssigkeitsgemisch überströmt. Zur Erzeugung der Flüssigkeitsgemische sowie definierter, pulsationsarmer Strömungsgeschwindigkeiten wurden Spritzenpumpen (Typ. C3000, Fa. TriContinent) eingesetzt.

Das genutzte Sensorlayout ist in Abb. 6 sowie in vergrößerter Ansicht mit in Blau angedeutetem Störkörper in Abb. 7 dargestellt.

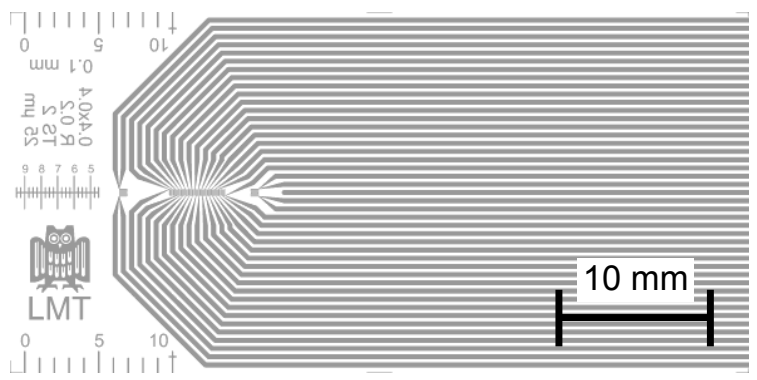

Abb. 6. Genutztes Sensorlayout. Der Sensor wird mittels eines FPC-Steckers kontaktiert.

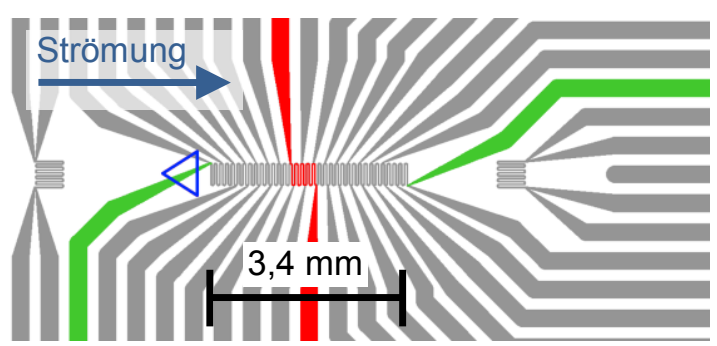

Abb. 7. Vergrößerte Ansicht des genutzten Sensorlayouts mit in Blau angedeutetem Störkörper. Die roten Leiterbahnen stehen für einen exemplarisch geheizten Bereich des Heizerarrays, die grünen Leitungen werden zusammen mit den restlichen Mäandern des Heizerarrays zur Widerstandsmessung genutzt.

Das Sensorlayout besteht aus drei mäanderförmigen Metallwiderständen, wobei der mittlere mit 16 jeweils halb überlappenden Bereichen mit je 0,4 x 0,4 $\mathrm{mm}^{2}$ Fläche, die einzeln aktiv geheizt werden können, das Heizerarray bildet. Aufgrund des geringen spezifischen Widerstandes der Aluminiumstrukturen sind alle Widerstände mittels Vierleitermessung angeschlossen, wodurch die Widerstände und somit die lokalen Temperaturen exakt bestimmt werden können. Der in Strömungsrichtung vor dem Störkörper gelegene Widerstand bietet die Möglichkeit, die Ausgangstemperatur des 
Mediums zu messen, um dadurch deren Einfluss auf die Viskosität berücksichtigen zu können.

Zur simultanen Messung der Strömungsgeschwindigkeit wurde bereits in vorherigen Arbeiten ein Laufzeitverfahren getestet. Hierfür wurde die Zeitdauer bestimmt, nach der ein in die Strömung eingeprägter und von ihr mitgeführter Wärmepuls am Ort eines in definiertem Abstand befindlichen Temperatursensors eine definierte Temperaturänderung hervorruft. Im Vergleich zur Hitzdrahtanemometrie erlaubt dieses Messverfahren die Bestimmung der Strömungsgeschwindigkeit unabhängig von den thermischen Eigenschaften der Flüssigkeit [5]. Im vorliegenden Fall soll hierfür der in Strömungsrichtung hinter dem Heizerarray angeordnete Temperatursensor genutzt werden. Durch Kenntnis der Strömungsgeschwindigkeit kann ihr Einfluss auf die Wirbelausdehnung bei der Bestimmung des Mischungsverhältnisses kompensiert werden.

Die realisierten Sensoren bestehen aus einer gesputterten, 0,5 $\mu \mathrm{m}$ dicken Aluminiumschicht auf einer $125 \mu \mathrm{m}$ dicken Kapton-Folie. Die Metallstrukturen wurden mittels einer $5 \mu \mathrm{m}$ dicken Polyamidimid-Schicht durch Spincoating unter Nutzung des Lacks Durimide 32A (Fa. Fujifilm) passiviert. Abb. 8 zeigt den fertigen Sensor. Die gemessenen Widerstände der einzelnen Heizerbereiche betragen bei $25^{\circ} \mathrm{C}$ je ca. $12 \Omega$, der Temperaturkoeffizient $\alpha_{0}$ bei der Referenztemperatur $0^{\circ} \mathrm{C}$ wurde in früheren Arbeiten zu 3,25 x $10^{-3} \mathrm{~K}^{-1}$ bestimmt [5].

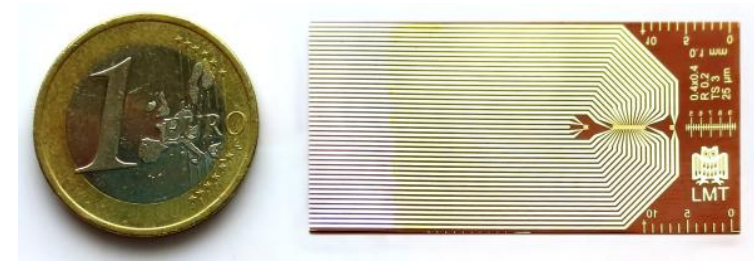

Abb. 8. Bild eines kompletten Sensors mit Aluminium-Mikrostrukturen auf Kapton-Folie.

\section{Messablauf}

Die Implementierung der Steuerung des Messablaufs erfolgte in LabVIEW (Fa. National Instruments). Für die ersten, orientierenden Messungen wurden vorab Gemische aus Methanol und deionisiertem Wasser mit definierten Volumenanteilen von $\phi=0,5$ und $10 \%$ hergestellt. Die Sensorfolie wurde derart in die Kammer eingebaut, dass sich zwischen dem ersten Heizer des Heizerarrays und dem Keil ein Abstand von ca. $0,1 \mathrm{~mm}$ ergab. Während der Messung wurde das Gemisch mit einer konstanten Flussrate in die Kammer eingeleitet und durchströmte den Einsatz, wodurch sich bei größeren Flussraten hinter dem Störkörper über dem Heizerarray ein stationäres Wirbelpaar bildete. Die getesteten Flussraten lagen zwischen 0,01 und $0,1 \mathrm{ml} / \mathrm{s}$, wodurch sich im Zuleitungskanal des Einsatzes vor dem Störkörper aufgrund der Querschnittsfläche von $1 \times 1 \mathrm{~mm}^{2}$ Strömungsgeschwindigkeiten zwischen 0,01 und $0,1 \mathrm{~m} / \mathrm{s}$ ergaben.

Die beiden separaten Temperatursensoren vor bzw. hinter dem Heizerarray wurden mit einer Leistung von $0,4 \mathrm{~mW}$ betrieben, wodurch sich auch ohne Strömung keine nennenswerte Eigenerwärmung zeigte. Während der vor dem Störkörper platzierte Temperatursensor kontinuierlich die Temperatur der Mischung erfasste, wurden die 16 Heizer des Arrays nacheinander entgegen der Hauptströmungsrichtung während einer Dauer von je $3 \mathrm{~s}$ mit einem konstanten Strom von ca. $65 \mathrm{~mA}$ beaufschlagt, was bei Raumtemperatur einer Heizleistung von je ca. $50 \mathrm{~mW}$ entspricht, die während der Heizphase aufgrund des Widerstandsanstiegs auf ca. $58 \mathrm{~mW}$ anstieg. Zu Beginn jedes Messzyklus wurde nach einer Pause von $5 \mathrm{~s}$ zur Stabilisierung des Wirbels der am weitesten hinter dem Störkörper gelegene Heizer für $5 \mathrm{~s}$ vorgeheizt. Dadurch sollte ausgeglichen werden, dass die folgenden, jeweils überlappenden Heizer immer zur Hälfte schon vom vorigen Heizer erwärmt sind, der erste jedoch nicht.

Um den aufgrund der Fertigung schwankenden Grundwiderständen Rechnung zu tragen, wurde der Heizstrom für jeden einzelnen der Heizerbereiche so eingestellt, dass bei Raumtemperatur die gleiche Heizleistung eingeprägt wird. Obwohl die betragsmäßig größeren Widerstände eine größere, absolute Widerstandsänderung mit der Temperatur aufweisen ist dadurch sichergestellt, dass alle Heizerbereiche bei gleicher Temperatur die gleiche Heizleistung erfahren. Zukünftig soll eine konstante Leistung eingeprägt werden, um die aufwändige Einzelkalibrierung zu vermeiden und reproduzierbarere Ergebnisse zu erzielen.

\section{Messergebnisse}

Abb. 9 zeigt die gemessenen Temperaturen des segmentweise entgegen der Strömungsrichtung durchgeschalteten Heizerarrays bei einer Flussrate von $0,01 \mathrm{ml} / \mathrm{s}$. Wie in Abb. 3 dargestellt, wird das Heizerarray durch den Störkörper von der Strömung abgeschattet, wobei die Strömungsgeschwindigkeit in größerer Entfernung zum Keil langsam wieder zunimmt und mit ihr auch der konvektive Wärmeabtransport steigt. Zusätzlich nimmt mit zunehmendem Methanolanteil der Wärmeeindringkoeffizient (die Wurzel aus dem Produkt von Wärmeleitfähigkeit, spezifischer Wärmekapazität und Dichte [9]) der Mischung ab, 
wodurch die Wärme schlechter vom Heizer abgeführt und somit eine höhere Heizertemperatur erreicht wird [10]. Die hierbei erreichte Empfindlichkeit der Heizertemperatur bzgl. des Methanolanteils liegt bei ca. 0,2 K/Vol.-\% und stimmt mit Ergebnissen früherer Arbeiten unter der Nutzung einzelner Mikroheizer in ruhender Flüssigkeit überein $[5,11]$.

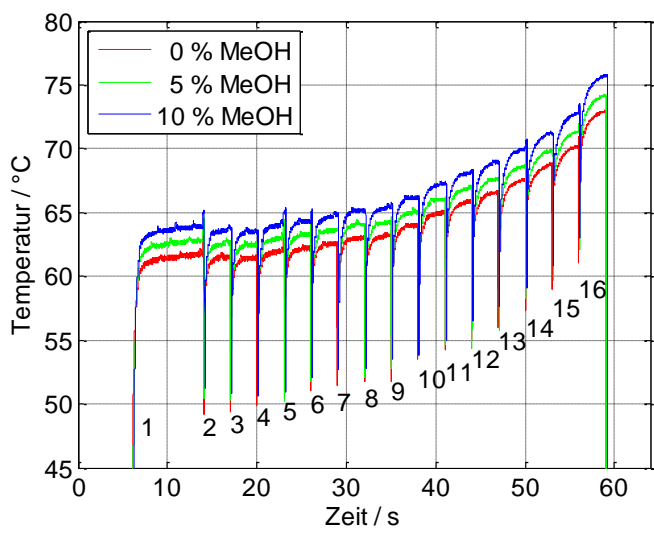

Abb. 9. Temperaturverlauf des Heizerarrays für verschiedene Methanolanteile bei einer Flussrate von 0,01 ml/s. Der Heizer Nummer 16 liegt am nächsten hinter dem Störkörper.

Abb. 10 zeigt den Temperaturverlauf des Heizerarrays in reinem Wasser bei unterschiedlichen Flussraten. Hier werden die Heizer in großer Entfernung zum Störkörper mit steigender Flussrate aufgrund der größeren erzwungenen Konvektion stärker gekühlt.

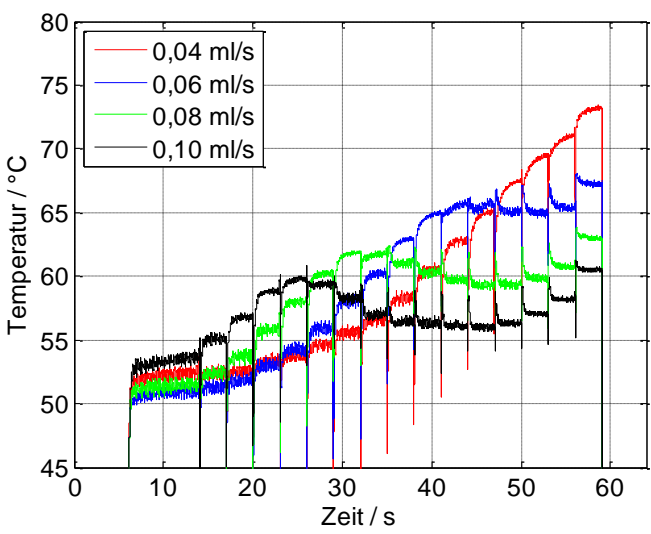

Abb. 10. Temperaturverlauf des Heizerarrays für reines Wasser bei verschiedenen Flussraten. An dem Heizer mit der höchsten Temperatur lässt sich die Ausdehnung des Wirbels erkennen.

Zudem bildet sich hinter dem Störkörper ab einer Flussrate von ca. 0,06 ml/s deutlich ein stehender Wirbel aus, wodurch der im Totgebiet liegende Teil des Heizerarrays in umgekehrter Strömungsrichtung überspült wird. An einer bestimmten Position ist somit die lokale Strömungsgeschwindigkeit über dem Heizerarray Null und die erzwungene Konvektion wird stark vermindert, wodurch der dort befindliche Heizer eine geringere Kühlung erfährt und sich dadurch stärker erwärmt.

In Abb. 11 ist der Temperaturverlauf des Heizerarrays bei einer Flussrate von $0,09 \mathrm{ml} / \mathrm{s}$ für verschiedene Methanolanteile in Wasser dargestellt.

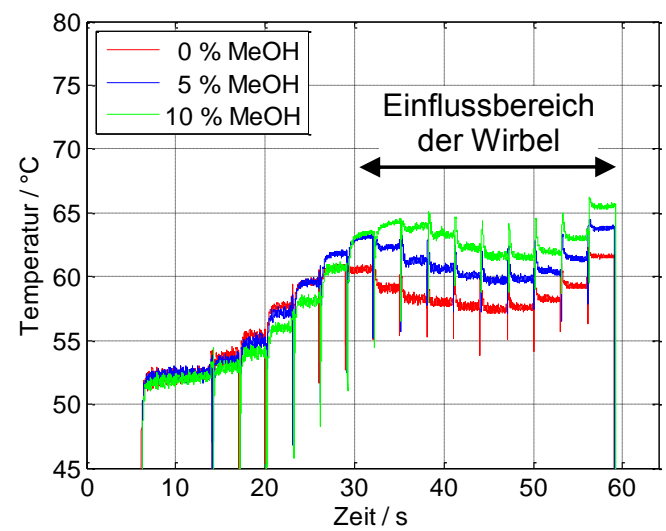

Abb. 11. Temperaturverlauf des Heizerarrays für verschiedene Methanolanteile bei einer Flussrate von $0,09 \mathrm{ml} / \mathrm{s}$.

Im Bereich weit hinter dem Störkörper ist kein Einfluss des Wirbels erkennbar. Der Einfluss des Methanols ist hier im Vergleich zur Messung bei geringer Flussrate (Abb. 9) sehr gering, da die Wärme hauptsächlich aufgrund erzwungener Konvektion abgeführt wird, welche bei der hohen Strömungsgeschwindigkeit nur wenig vom Methanolanteil beeinflusst ist. In Richtung des Störkörpers nimmt die Temperatur der einzelnen Heizerbereiche aufgrund der abnehmenden Strömungsgeschwindigkeit zu und erreicht an der Stelle des Wirbelendes ein Maximum. Dieses Maximum verschiebt sich mit steigendem Methanolanteil entgegen der Strömungsrichtung aufgrund der zunehmenden kinematischen Viskosität (siehe Abb. 2), die infolge der abnehmenden Reynoldszahl (siehe GI. 2) zu einer Verringerung der Wirbelausdehnung führt [1]. Näher am Störkörper liegt die vom Wirbel beeinflusste Zone, in der die Strömungsgeschwindigkeit zunächst zunimmt und am Störkörper schließlich wieder verschwindet, wodurch auch die Heizertemperaturen ein lokales Minimum zeigen. In dieser Zone beträgt die Empfindlichkeit des Sensors, d.h. die Änderung der Heizertemperatur in Abhängigkeit vom Methanolanteil, ca. 0,5 bis $0,6 \mathrm{~K} / \mathrm{Vol} .-\%$ und ist damit nahezu verdreifacht gegenüber den Messungen in ruhender Flüssigkeit.

In ersten Messungen zur Reproduzierbarkeit des Messprinzips ergab sich in jeweils drei aufeinanderfolgenden Messungen eine Schwankung der gemessenen Temperaturen 
von weniger als $\pm 0,25 \mathrm{~K}$. Dies resultiert im Zusammenhang mit der gemessenen Empfindlichkeit in einer möglichen Auflösung des Methanolanteils von derzeit ca. 0,5 Vol.-\%, die es in weiteren Messungen noch zu bestätigen gilt. Zudem sollte durch die inhärente mehrfache Messung eine Mittelung und damit eine höhere Genauigkeit erzielbar sein.

\section{Fazit und Ausblick}

Ein neues Sensorprinzip zur Bestimmung des Mischungsverhältnisses von binären Flüssigkeitsgemischen wurde erarbeitet, welches auf stationären Wirbelpaaren hinter einem in eine Strömung eingebrachten Störkörper basiert. Durch die verschiedenen kinematischen Viskositäten der Ausgangsstoffe, die in einer vom Mischungsverhältnis abhängigen Gesamtviskosität resultieren, wird die Ausbreitung des Wirbelpaares in Strömungsrichtung vom Mischungsverhältnis beeinflusst. Diese Ausdehnung wurde unter Nutzung eines mikrostrukturierten Heizerarrays aus Dünnschicht-Metallwiderständen bestimmt, wodurch die Messung des Mischungsverhältnisses von Methanol/ Wasser-Gemischen möglich war. Bei höheren Strömungsgeschwindigkeiten wurde nahezu eine Verdreifachung der Empfindlichkeit gegenüber früheren Arbeiten erreicht.

Als nächster Schritt muss die Ansprechzeit des Sensorprinzips auf Änderungen des Mischungsverhältnisses untersucht und optimiert werden. Gerade bei diesem Messprinzip sind hier Nachteile aufgrund des Totgebiets hinter dem Störkörper zu erwarten, die es zu quantifizieren gilt.

Darüber hinaus soll der in Strömungsrichtung hinter dem Heizerarray gelegene Temperatursensor in zukünftigen Messungen genutzt werden, um simultan die Strömungsgeschwindigkeit bestimmen zu können. Zusätzlich soll der Einfluss der Umgebungstemperatur untersucht werden. So verringert sich bei steigender Temperatur der Einfluss des Methanolanteils auf die kinematische Viskosität, was in einer Verringerung der Empfindlichkeit des Messprinzips resultieren sollte (siehe Abb. 2). Beide Effekte müssen für eine genaue Bestimmung des Mischungsverhältnisses berücksichtigt werden. Insgesamt muss eine geeignete Mess- und Auswertemethode entwickelt werden, um möglichst rasch und genau das Mischungsverhältnis zu bestimmen.

Des Weiteren zeigten Blasen in der Messkammer einen gravierenden Einfluss auf das Messergebnis, da sie die Strömungsverhältnisse stark beeinflussten. Somit muss in weiteren Arbeiten ein verstärkter Fokus auf die Vermeidung von in der Kammer anhaftenden Blasen bzw. ein strömungsoptimiertes Kammerdesign gelegt werden. Zudem muss das Messprinzip gerade im Hinblick auf den beabsichtigten Einsatz in Direktmethanolbrennstoffzellen für den mobilen Einsatz auf den Einfluss von Vibrationen untersucht werden, die im Betrieb zu erwarten sind.

\section{References}

[1] C. K. Dyer, Fuel cells for portable applications, Journal of Power Sources 106, 31-34 (2002); doi: 10.1016/S0378-7753(01)01069-2

[2] M. Nölke, Entwicklung eines Direkt-MethanolBrennstoffzellensystems der Leistungsklasse kleiner $5 \mathrm{~kW}$, Jülich, Forschungszentrum Jülich (2007); ISBN-10: 3893364811

[3] A. Scott, W. Taama, J. Cruickshank, Performance of a direct methanol fuel cell, Journal of Applied Electrochemistry 28, 289-297 (1998), doi: 10.1023/A:1003263632683

[4] W. P. Trautwein, AdBlue als Reduktionsmittel für die Absenkung der $\mathrm{NO}_{x}$-Emissionen aus Nutzfahrzeugen mit Dieselmotor, DGMKForschungsbericht 616-1, Deutsche Wissenschaftliche Gesellschaft für Erdöl, Erdgas und Kohle e.V. Hamburg (2003); ISBN-10: 393641808X

[5] B. Schmitt, C. Kiefer, A. Schütze, Microthermal sensors for determining fluid composition and flow rate in fluidic systems, Proc. SPIE 8763 (2013); doi: 10.1117/12.2017293

[6] W. H. Hucho, Aerodynamik der stumpfen Körper, 2. Auflage, Vieweg+Teubner, Springer Wiesbaden (2011); ISBN-10: 3834814623

[7] H. R. Tränkler, E. Obermeier, Sensortechnik Handbuch für Praxis und Wissenschaft, Springer Verlag (1998); ISBN-10: 3540586407

[8] S. Z. Mikhail, W. R. Kimel, Densities and Viscosities of Methanol-Water Mixtures, Journal of Chemical and Engineering Data 6, 533-537 (1961); doi: 10.1021/je60011a015

[9] P. von Böckh, Wärmeübertragung - Grundlagen und Praxis, zweite, bearbeitete Auflage, Springer Verlag (2006); ISBN-10: 3540314326

[10] Y. He, Rapid thermal conductivity measurements with a hot disk sensor: Part 1. Theoretical considerations, Thermochimica Acta 436, 122129 (2005); doi: 10.1016/j.tca.2005.06.026

[11] B. Schmitt, C. Kiefer, A. Schütze, Mikrothermischer Sensor zur Bestimmung von Methanolkonzentration und Strömungsgeschwindigkeit für Direktmethanolbrennstoffzellen,

MikroSystemTechnik-Kongress 2013, Aachen, 14.-16. Oktober 2013. 